\title{
Relief of Chronic Shoulder and Neck Pain by Electro-Acupuncture and Transcutaneous Electrical Nervous Stimulation: A Randomized Crossover Trial
}

\author{
Madoka Yoshimizu, MPH, Alan R. Teo, MD, ${ }^{2}$ Masahiko Ando, MD, \\ Kosuke Kiyohara, $\mathrm{DPH}^{1}$, and Takashi Kawamura, $\mathrm{MD}^{1}$
}

\begin{abstract}
Background: Chronic neck and shoulder pain is common and disabling.

Objective: The aim of this study was to compare the effectiveness of electro-acupuncture and transcutaneous electrical stimulation (TENS) for relief of shoulder and neck pain.

Materials and Methods: Design: This was a randomized crossover trial. Subjects: Ninety patients were enrolled, with a mean age of 34 years, and with females slightly outnumbering males. All subjects completed the study. Intervention: For electro-acupuncture, acupuncture needles were placed in four different acupoints in the trapezius muscle and each subject underwent a 15-minute session of low-frequency electrical stimulation. TENS treatment was similar and used as an active comparator, with a 2-week washout period between treatments. Outcome Measures: The primary outcome was reduction in pain as measured by a $100 \mathrm{~cm}$ visual analogue scale. Secondary outcomes included quality-of-life (QoL) measures.

Results: Electro-acupuncture produced significantly greater reduction in pain than TENS did the first 2 days after treatment ( $p=0.001$ and $p=0.003$, respectively), with pain decreasing from 56 to 33 and 34 versus from 55 to 42 and 42. Electro-acupuncture also produced a significant improvement in the vitality subscale of the Short Form-36. No adverse effects or carryover effect were detected.

Conclusions: The results of this study offer preliminary evidence for the comparative effectiveness of electroacupuncture over TENS for the acute relief of chronic shoulder and neck pain in adults.
\end{abstract}

Key Words: Electro-Acupuncture, Transcutaneous Electrical Nerve Stimulation, Crossover Trial, Neck Pain, Shoulder Pain

\section{INTRODUCTION}

$\mathbf{S}$ HOULDER AND NECK COMPLAINTS are extremely common in developed countries. A Swedish study of combined neck and shoulder pain estimated a prevalence of $18 \%$ in a random adult population. ${ }^{1}$ A large French study of a working population found the prevalence of chronic neck and shoulder pain in women was $15 \%-18 \%$ and $8 \%-10 \%$ in men. ${ }^{2}$ Health surveys in Japan have found shoulder-neck to be the most common physical complaint affecting $13 \%$ of the population. ${ }^{3}$ Such complaints cause a significant disease burden, including chronic disability, diminished work productivity, and decreased ability to perform activities of daily living. ${ }^{4-6}$ Shoulder-neck pain is an extremely broad term, but careful studies of more precise descriptors have shown that, by far, the most common form of pain is a dull muscle

\footnotetext{
${ }^{1}$ Kyoto University Health Service, Kyoto, Japan.

${ }^{2}$ Robert Wood Johnson Foundation Clinical Scholars Program, University of Michigan, Ann Arbor, Michigan.
} 
ache (pain that is like being stiff after exercise), affecting nearly $90 \%$ of patients with persistent pain. ${ }^{7}$ In Japan, the single word katakori encompasses this quality of pain in the neck and shoulder, which is essentially a myofascial pain in a region extending from the posterior neck ( $\mathrm{C}-1$ level) through the shoulders and as low as the inferior scapula (T-7 level). Patients may describe the pain as stiffness, discomfort, or an ache.

Patients in pain often seek complementary and alternative medicine treatment, such as acupuncture. In 1994, 1,000,000 Americans utilized acupuncture, ${ }^{8}$ which doubled to $>2,000,000$ by 2002 ; $>4 \%$ of Americans report lifetime use. ${ }^{9}$ Among Japanese patients with chronic neck and shoulder pain, $59 \%$ of people are treated by acupuncturists and chiropractors, far more commonly than by Western medical practitioners. ${ }^{3}$ Two such interventions that have shown some evidence of success include electro-acupuncture and transcutaneous electrical nerve stimulation (TENS). ${ }^{10-13}$ Both modalities involve the conduction of low frequency electricity; in the former, the conduction is subcutaneous through typical acupuncture needles, while, in the latter, it is through non-penetrating electrode pads. Nonetheless, highquality evidence of the efficacy of these treatments is scarce. Green and colleagues, writing for the Cochrane Collection, noted that few randomized controlled trials (RCTs) have been conducted on acupuncture for shoulder pain, those that exist are methodologically diverse with poor descriptions of their interventions, and, thus, the researchers concluded that there is a lack of evidence regarding whether acupuncture works or, conversely, is harmful. ${ }^{14}$ To the current authors' knowledge, there has never been an RCT trial in the English-language literature comparing the effectiveness of electro-acupuncture and TENS for shoulder and neck pain. Trials that have compared electro-acupuncture to TENS have focused on related conditions such as lumbago; ${ }^{10-12,15,16}$ and trials that have examined neck and shoulder pain have generally compared acupuncture to a placebo (sham). ${ }^{4,5,17,18}$ Moreover, there has been a call for more RCTs that mirror the more-realistic clinical situation in which a clinician must choose between two treatment options, rather than one versus nothing (i.e., placebo). ${ }^{16,19}$

Thus, the aim of this clinical trial was to provide a practical, head-to-head comparison of the efficacy of electro-acupuncture and TENS for adults who have chronic shoulder and neck pain or stiffness, using a randomized crossover trial design.

\section{MATERIALS AND METHODS}

\section{Study Design}

This study used a prospective, two-period, two-treatment crossover design with patients randomized to one of the following two treatment sequences: electro-acupuncture followed by TENS; or TENS followed by electro-acupuncture. Because patients received two distinct active treatments making masking unfeasible, this study was conducted as an open trial.

\section{Patients}

Patients between the ages of 20 and 65 who self-identified (as confirmed by written history) as having chronic pain in the neck and/or shoulder region and had minimal experience with acupuncture or TENS were eligible for inclusion. Patients were recruited from the university community, including both students and staff. The clinical definition used for neck-shoulder pain (i.e., katakori) was "tightness or stiffness in the shoulder and lower neck, especially the trapezius and semispinalis muscles." Patients were excluded if they met any of the following criteria: (1) concurrently undergoing regular (one or more times a week) treatment for neck-shoulder pain; (2) fear of acupuncture techniques; (3) history of a neurologic condition; (4) history of a significant orthopedic condition; and (5) any other factor that would impair involvement in a clinical trial (e.g., inability to complete the follow-up). Baseline characteristics were collected for age, gender, history of myofascial pain, related symptoms, and past medical history. Patients were allocated to one of two groups using block randomization with a block size ranging from 2 to $6 .^{20}$ Randomization was performed by a researcher who was uninvolved with the interventions and data analysis. This study was approved by the ethics committee of the Kyoto University Faculty of Medicine, and written informed consent was obtained from all patients before enrollment. A total of 90 patients were enrolled, with a mean age of 34 years, and females slightly outnumbered males.

\section{Interventions}

Interventions were performed with patients in a prone position in an examination room at the Kyoto University Health Service. No special environmental interventions, including aromatics, music, or lighting, were used. A single licensed acupuncturist (M.Y.), with 5 years of professional experience and trained in both modalities, performed all treatments. For the electro-acupuncture treatment, four $0.20 \times 50-\mathrm{mm}$ stainless-steel, disposable acupuncture needles (Yamashō ${ }^{\circledR}$ NEO, Nagahama-shi, Shiga-ken, Japan) were inserted at four sites in the upper back and shoulder of each subject. ${ }^{18}$ Japanese-style acupuncture was used. This style has two major distinctions from Chinese acupuncture: first, a technique of acupuncture needle insertion called kanshinhou utilizes a hollow tube through which the needle is guided; and second, Japanese acupuncture needles are shorter $(3-60 \mathrm{~mm})$ and thinner $(0.16-0.24 \mathrm{~mm})$ than Chinese needles.

The clinician first palpated for four acupoints associated with neck-shoulder pain and positioned in the trapezius: the left and right Jianjing (GB 21) and Jianwaishu (SI 14). For 
electro-acupuncture, needles were then inserted into the Ah Shi point within a $1-\mathrm{cm}$ radius of these acupoints. Needles were inserted perpendicular to the skin, and not twirled. Both acupuncture points and Ah Shi points have been shown to have a high degree (71\%) of correspondence. ${ }^{21}$ Consistent with previous research protocols and actual clinical practice, needles were inserted into muscle tissue to a depth of between 10 and $15 \mathrm{~mm} .{ }^{16,22}$ The needles were then connected to a low-frequency electrical generator (Techno Link ${ }^{\circledR}$ Techtron DSP, Niigata-shi, Niigata-ken, Japan) set to an electrical frequency of $0.5-10 \mathrm{~Hz}$, a current of $4-4.1 \mathrm{~mA}$, and a resistance of $500 \Omega$ (ohms); then, the patients underwent 15 minutes of stimulation. This duration was chosen based on standard clinical practice. ${ }^{23}$ Electrical strength was adjusted to the highest level that each patient could tolerate comfortably (typically creating muscle contraction) and readjusted after the first 5 minutes. For the TENS treatment, patients had four gel-type electrode pads placed at the same points (bilateral GB 21 and SI 14), using the same electrical generator set to an electrical frequency of 1$1.5 \mathrm{kHz}$, a current of 60-63 mA, and a resistance of $500 \Omega$ (ohms). Duration of stimulation and adjustment were the same as with electro-acupuncture.

Participants received a single treatment session for each intervention. There was a 2-week washout period before the second randomized treatment but no washout prior to the first. Any subject who developed an adverse reaction was treated appropriately and study treatment was stopped. Patients continued taking their routine medications for any chronic medical conditions (e.g., antihypertensives), but were instructed to abstain from taking any new medications (over-the-counter or prescription), including analgesics, during the study period.

\section{Outcome Measures}

Previous articles have commented on the difficulty of establishing objective outcome measures for myofascial pain syndromes, but from the patient's perspective (which, after all, is the most clinically relevant), subjective functional improvement is often measured. ${ }^{24}$ Thus, the primary outcome for this study was pain relief as measured by a 100-mm visual analogue scale (VAS), ranging from 0 ("no pain at all") to 100 ("worst neck and shoulder pain I have experienced"). Subjects drew a hash mark at the point along the line that best represented their pain level at the time referenced in the question. Patients were asked to provide VAS scores for a total of eight timepoints per intervention: immediately before and after treatment and once daily on the second through seventh day after treatment. To exclude recall bias, patients completed separate questionnaires at each of these timepoints.

Secondary outcome variables included QoL measures and safety. QoL was assessed using a subset of 15 questions derived from the Short Form (SF-36) Japanese, version $2 .^{25}$
Four of the subscales in the SF-36 acute form (symptoms over the last week) were utilized: (1) role physical (4 items); (2) bodily pain (2 items); (3) vitality (4 items); and (4) mental health (5 items). These scores were transformed linearly to range from 0 (worst score) to 100 (best score). Patients provided answers twice per intervention, once before treatment and once per week afterward. Safety of the treatment was assessed by report of adverse events, and by monitoring of blood pressure (BP) and heart rate (HR) before and after each intervention.

\section{Statistical Analysis}

The average reduction in pain was calculated as the mean difference between the VAS score immediately before treatment and those from immediately after through 6 days after treatment. A total of 90 subjects (45 for each group) were planned to be accrued into this study, which assured at least $90 \%$ statistical power to detect a $4.5-\mathrm{mm}$ difference in the average VAS score at a 5\% significance level. The improvement of QoL was calculated as the difference between pretreatment and post-treatment QoL scores. The effect on vital signs was also evaluated based on the pretreatment and post-treatment values. Analyses were performed on an intention-to-treat (ITT) basis.

Given that the main potential confounder in a crossover trial is treatment-period interactions, that is, carryover effects, ${ }^{26}$ the carryover effect was first assessed using an unpaired $t$-test applied to the individual sums of the first- and the second-period data. Treatment effects and period effects were then assessed using an unpaired $t$ test according to standard analytical methods. ${ }^{27}$ In multivariate analyses, analyses of covariance were used for a crossover design by the MIXED procedure of SAS, version 9, software (SAS Institute, Cary, NC). When a treatment-period interaction term was not statistically significant, the interaction term was deleted and the reduced model was used including pretreatment value, gender, age, treatment, and period. All tests of significance were two-sided and $p<0.05$ was defined as significant.

\section{RESULTS}

\section{Study Population}

Patient flow through the study is illustrated in Figure 1. Ninety patients were enrolled in the study from September 2005 through November 2006 and all completed the planned treatment. According to the randomization procedure, 45 patients were allocated to each group. Subjects in group A received electro-acupuncture followed by TENS, while those in group B received TENS followed by electroacupuncture. Because of a technical error, two group A patients underwent the group B protocol, but were analyzed on an ITT basis. 


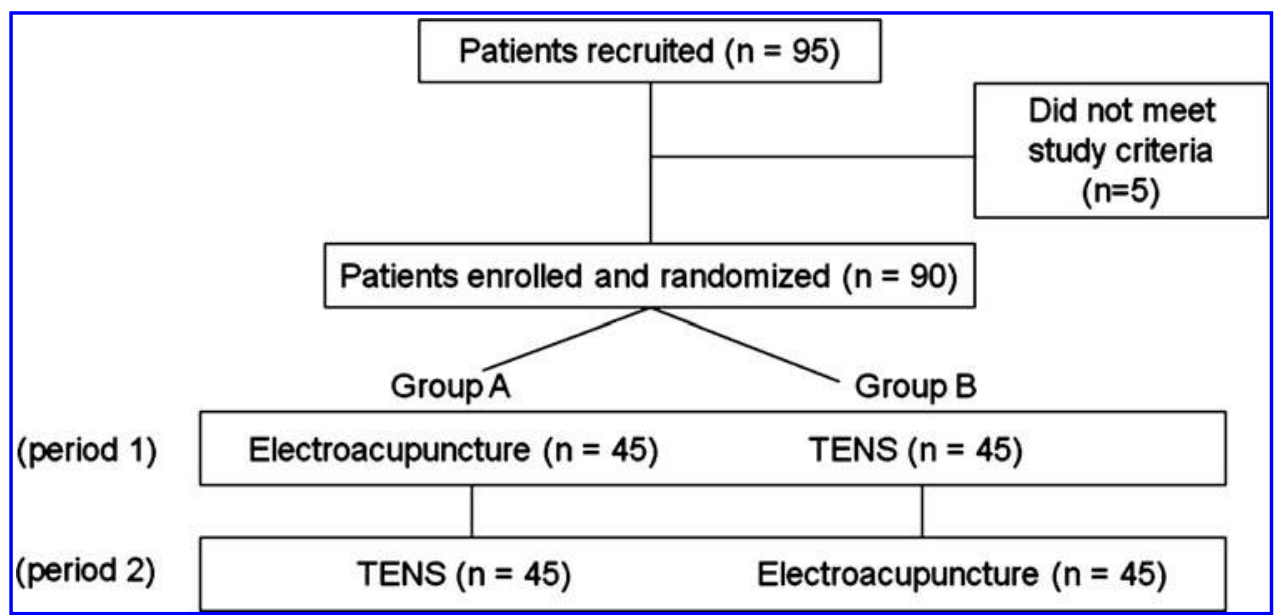

FIG. 1. Outline of patient enrollment and randomization. TENS, transcutaneous electrical stimulation.

Baseline characteristics of the study patients are shown in Table 1. The mean age was 34 years and females slightly outnumbered males. Most patients complained of pain or stiffness in both shoulders and in the neck, and these patients' symptoms were chronic.

\section{Reduction in Pain}

Shoulder and neck pain over time by treatment is shown in Figure 2. Patients had moderate pain at baseline (a VAS of 55 for TENS and a VAS of 56 for electro-acupuncture), which substantially decreased immediately after treatment in both treatment arms (VAS of 34 for both TENS and

Table 1. Baseline Characteristics of Patients

\begin{tabular}{lcc}
\hline & $\begin{array}{c}\text { Group A } \\
\text { Electro-acupuncture } \\
\text { followed by } \\
\text { TENS } n=45\end{array}$ & $\begin{array}{c}\text { Group B } \\
\text { TENS followed } \\
\text { by Electro- } \\
\text { acupuncture } n=45\end{array}$ \\
Characteristics & $33 \pm 11$ & $34 \pm 12$ \\
\hline Age, yr \pm SD & $25 / 20$ & $27 / 18$ \\
Female/male & & \\
Previous acupuncture & $1(2 \%)$ & $1(2 \%)$ \\
for shoulder/neck & & \\
symptom & & \\
for other complaints & $4(9 \%)$ & $8(4 \%)$ \\
Pain and stiffness location, no. $(\%)$ & $1(2 \%)$ \\
Shoulder & $14(31 \%)$ & $37(80 \%)$ \\
Neck & $1(2 \%)$ & \\
Both & $30(67 \%)$ & $7(16 \%)$ \\
Duration of symptoms, no $(\%)$ & $11(24 \%)$ \\
$<1$ year & $8(18 \%)$ & $11(24 \%)$ \\
1-4 years & $13(28 \%)$ & $4(9 \%)$ \\
5-9 years & $9(20 \%)$ & \\
10-19 years & $12(27 \%)$ & \\
$>$ 20 years & $3(7 \%)$ &
\end{tabular}

TENS, transcutaneous electrical nerve stimulation; yr, years; SD, standard deviation. electro-acupuncture). Electro-acupuncture then produced sustained pain reduction on days 2 and 3 (VAS scores of 33 and 34, respectively), whereas TENS produced a morerapid decay in effect (VAS scores of 42 on days 2 and 3). Electro-acupuncture provided significantly more relief of pain, compared to TENS on days 2 and 3 ( $p=0.001$ and $p=0.003$, respectively).

In the standard crossover-design analyses, there was neither a significant carryover effect $(p=0.508)$ nor a period effect ( $p=0.108$ ) on pain reduction. Because there was no evidence of systematic bias caused by order of treatment, pooled data from both periods were used to estimate treatment effect. For the treatment effect, which was assessed using the average reduction in pain from immediately after treatment through day 7, electro-acupuncture showed a further 5.3-mm improvement in VAS, score compared with TENS $(p=0.025)$. In an analysis of covariance, the treatment-period interaction term was not significant $(p=0.296)$.

The pain-relief effect of electro-acupuncture remained significantly greater $(p=0.010)$ after adjustment for pretreatment VAS score, gender, age, and period. No covariates,

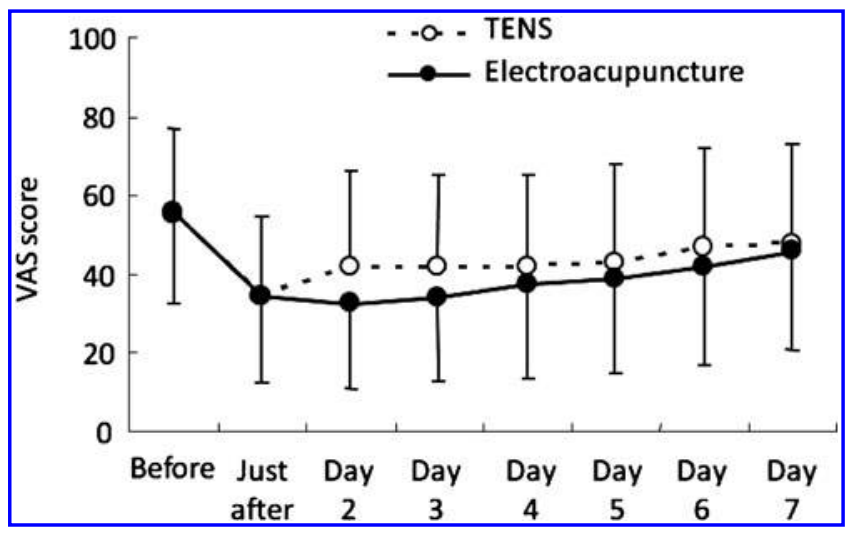

FIG. 2. Shoulder and neck pain over time by treatment. VAS, visual analogue scale; TENS, transcutaneous electrical stimulation. 
Table 2. Quality of Life Measurements Before and After Treatment

\begin{tabular}{|c|c|c|c|c|c|}
\hline \multirow[b]{2}{*}{ Subscale of SF-36 } & \multicolumn{2}{|c|}{ Electro-acupuncture } & \multicolumn{2}{|c|}{ TENS } & \multirow[b]{2}{*}{$p$-Value* } \\
\hline & Before & After & Before & After & \\
\hline Role physical & $83.3 \pm 19.2$ & $89.0 \pm 14.8$ & $84.2 \pm 19.4$ & $86.1 \pm 17.4$ & 0.129 \\
\hline Bodily pain & $67.5 \pm 23.0$ & $72.5 \pm 21.4$ & $66.7 \pm 23.2$ & $68.4 \pm 20.7$ & 0.314 \\
\hline Vitality & $54.2 \pm 21.1$ & $61.5 \pm 18.1$ & $56.9 \pm 19.5$ & $58.6 \pm 21.7$ & 0.005 \\
\hline Mental health & $70.9 \pm 17.6$ & $72.3 \pm 17.3$ & $70.3 \pm 20.5$ & $70.6 \pm 20.2$ & 0.572 \\
\hline
\end{tabular}

SF-36, Short-Form-36; TENS, transcutaneous electrical nerve stimulation.

*Comparison was made for the pre- and post-treatment difference between electroacupuncture and TENS.

except pretreatment VAS scores, were significantly correlated with pain reduction.

\section{QoL}

QoL measures showed that vitality was similar pretreatment (54.2 for electro-acupuncture and 56.9 for TENS), but that QoL only improved post-treatment for electroacupuncture (61.5) and not for TENS (56.6). In the analysis of covariance, electro-acupuncture produced a significantly greater improvement in vitality $(p=0.005)$ than TENS did after adjustment for pretreatment score, gender, age, and period (Table 2). For role physical, bodily pain, and mental health subscales, there were no significant differences in improvement between electro-acupuncture and TENS. The treatment-period interaction term was not significant in these analyses. Furthermore, there were no significant carryover effects in the role physical, bodily pain, vitality, and mental health subscales of the SF-36 $(p=0.741,0.646$, 0.273 , and 0.072 , respectively).

\section{Safety}

No serious adverse events were reported. BP and HR were stable for both electroacupuncture and TENS (Table 3). There were no significant carryover effects in systolic BP, diastolic BP, and HR ( $p=0.213,0.189$, and 0.825 , respectively). Systolic and diastolic BP readings were similar between electro-acupuncture and TENS, and no significant treatment effects ( $p=0.307$ and 0.312 , respectively) were found. In terms of HR, a slightly increased pretreatment value in electroa-cupuncture, probably because of a fear of pricking pain, decreased to the same level as TENS after treatment, yielding a small, but significant treatment-related change $(p=0.021)$. In the analysis of covariance, electro-acupuncture was associated with a significantly larger change in HR $(p=0.021)$ than TENS after adjustment for pretreatment score, gender, age, and period. There were no such differences in systolic or diastolic BP between electro-acupuncture and TENS in the analysis of covariance. The treatment-period interaction term was not significant in either of these analyses.

\section{DISCUSSION}

To the authors' knowledge, this is the first randomized trial that compared electro-acupuncture and TENS for shoulder and neck pain. The results suggest that both electro-acupuncture and TENS are effective short-term therapies for chronic shoulder and neck pain, but electroacupuncture is preferable because its pain-relieving effect is more durable. Specifically, the superiority of electroacupuncture continued for at least 2 days after treatment and then gradually attenuated.

The effect size of electro-acupuncture, while not large, was clinically significant. Specifically, electro-acupuncture produced a $41 \%$ reduction in pain 1 day after treatment, whereas TENS produced only a $24 \%$ reduction at that same timepoint. For patients with chronic pain, a $16 \%$ benefit for a treatment modality is meaningful and similar to results

Table 3. Vital Signs Before and After Treatment

\begin{tabular}{|c|c|c|c|c|c|}
\hline \multirow[b]{2}{*}{ Vital sign } & \multicolumn{2}{|c|}{ Electro-acupuncture } & \multicolumn{2}{|c|}{ TENS } & \multirow[b]{2}{*}{$p$-Value* } \\
\hline & Before & After & Before & After & \\
\hline \multicolumn{6}{|c|}{ Blood pressure } \\
\hline Systolic & $109.4 \pm 16.4$ & $109.0 \pm 14.9$ & $110.0 \pm 14.5$ & $108.6 \pm 14.4$ & 0.307 \\
\hline Diastolic & $66.4 \pm 13.7$ & $67.5 \pm 11.8$ & $66.2 \pm 13.1$ & $66.0 \pm 13.7$ & 0.312 \\
\hline Heart rate & $66.2 \pm 9.8$ & $62.6 \pm 7.9$ & $64.9 \pm 8.7$ & $62.5 \pm 8.0$ & 0.021 \\
\hline
\end{tabular}

TENS, transcutaneous electrical nerve stimulation.

*Comparison was made for pre- and post-treatment difference between electroacupuncture and TENS. 
found in other studies, such as in acupuncture for low-back pain. ${ }^{28}$ Improvement in vitality was more modest, at $13 \%$ for electro-acupuncture versus $-1 \%$ for TENS; we suspect this was the result of post-treatment measurement not occurring until 1 week later.

The relative simplicity and uniformity of the electroacupuncture treatment protocol makes it amendable to both clinical application and reproducibility. Only four needle locations, standardized to easily identifiable anatomical landmarks on the easily accessible trapezius muscle, with just 15-minute electrical stimulation was enough to produce both a clinically and statistically significant difference. Physicians and researchers have noted that the efficacy of acupuncture may depend on the individual skill of the practitioner, and interpatient variation in placement of needles is widely regarded by acupuncturists as essential to treatment. Electro-acupuncture requires less technical expertise than manual acupuncture, because even a deviation off an acupoint is partially accommodated for by the regional effect of the electrical current. With relatively limited training, even non-acupuncturist clinicians working in primary care or pain clinics could be capable of performing the electroacupuncture treatment protocol used in this study.

Strengths of this study included its randomized design, perfect follow-up rate, lack of interoperator bias, and relatively larger sample size (especially when considering the effective doubling of data with a crossover design), compared to similar studies. A crossover design was well-suited to this trial for two reasons. One, treatment for myofascial neck and shoulder pain temporarily alleviates but does not cure the pain, which is key to achieving a washout between interventions. ${ }^{26,27}$ Second, this study uniquely allowed patients to serve as their own controls, which is useful when treatment responses and subjective assessments of improvement have wide individual variations.

Another major strength of this study is its clinical applicability. Designed to be a practical comparison, this study was a head-to-head comparison of two reasonable, comparable therapeutic options used in clinical practice. Despite neck and shoulder complaints being among the most prevalent in primary care, few high-quality studies have compared the results of the many treatment choices available. By suggesting the superiority of an acupuncture technique to a technique similar in all ways, except for use of needling, this study helps fill in the gap of knowledge necessary for clinicians to make good, evidence-based treatment decisions.

Because this was an open trial, a placebo effect could have accounted for observed differences. However, the authors find this unlikely for two reasons. First, the pain trend observed in this study argues against a simple placebo effect. TENS provided significant immediate relief; indeed, pain relief from TENS was essentially identical to electro-acupuncture immediately after treatment. This trend changed, however, a day after treatment when electro-acupuncture showed that it was more effective. If subjects improved merely because of an anticipatory effect, it would be quite odd for this delayed peak in the effectiveness of acupuncture, but it is consistent with acupuncturists' clinical experience. In short, the authors suggest that the relief from TENS served as an effective control intervention. Second, both interventions were given equal consideration with equal one-on-one therapeutic care, which should have equalized any psychological benefits intrinsic to undergoing treatment. The authors felt that it was not feasible to create a believable placebo treatment group with electro-acupuncture. Moving needle placement away from acupoints, though used sometimes in sham manual acupuncture, was also felt to be too similar to real treatment because the larger area of effect provided by electrical stimulation.

This study has some other noteworthy limitations. First, because it did not include a placebo control, it was not possible to evaluate the magnitude of change relative to no treatment at all in this study population. Second, lack of blinding limited the internal validity of the study. Third, treatment was limited to a single session for each modality, whereas in actual clinical practice most patients would undergo repeated treatments. Studies with longer treatment and follow-up periods to evaluate how long-lasting a benefit can be achieved are warranted. ${ }^{29,30}$ Fourth, outcomes reflected subjective data, based on a VAS and the SF-36. In future experiments, adding more physiological measures as well as use of validated tools, such as a pressure algometer, ${ }^{29,30}$ would complement subjective measures.

\section{CONCLUSIONS}

Taken together, these results provide preliminary evidence supporting the use of electro-acupuncture for relief of neck and shoulder pain. It is notable that a single, simple, and short form of electro-acupuncture treatment can make a significant reduction of chronic symptoms, compared to TENS treatment. More research, including longitudinal follow-up, a placebo treatment arm, and other outcome measures are essential to strengthen and validate these findings.

\section{ACKNOWLEDGMENTS}

Dr. Teo wishes to acknowledge support for this study provided by grants from the Robert Wood Johnson Foundation Clinical Scholars program and the University of California, San Francisco School of Medicine Office of International Programs.

\section{DISCLOSURE STATEMENT}

No competing financial interests exist. 


\section{REFERENCES}

1. Westerling D, Jonsson BG. Pain from the neck-shoulder region and sick leave. Scand J Soc Med. 1980;8(3):131-136.

2. Cassou B, Derriennic F, Monfort C, Norton J, Touranchet A. Chronic neck and shoulder pain, age, and working conditions: longitudinal results from a large random sample in France. Occup Environ Med. 2002;59(8):537-544.

3. Editors of the Health and Welfare Statistics Association. Trends in National Health and Welfare [in Japanese]. Tokyo: Health and Welfare Statistics Association; 2004.

4. Guerra de Hoyos JA, Andrés Martín Mdel C, Bassas y Baena de Leon E, Vigára Lopez M, Molina López T, Verdugo Morilla FA, González Moreno MJ. Randomised trial of long term effect of acupuncture for shoulder pain. Pain. 2004; 112(3):289-298.

5. He D, Veiersted KB, Høstmark AT, Medbø JI. Effect of acupuncture treatment on chronic neck and shoulder pain in sedentary female workers: a 6-month and 3-year follow-up study. Pain. 2004;109(3):299-307.

6. Rempel DM, Harrison RJ, Barnhart S. Work-related cumulative trauma disorders of the upper extremity. JAMA. 1992; 267(6):838-842.

7. Brattberg G, Thorslund M, Wikman A. The prevalence of pain in a general population: The results of a postal survey in a county of Sweden. Pain. 1989;37(2):215-222.

8. Paramore LC. Use of alternative therapies: estimates from the 1994 Robert Wood Johnson Foundation National Access to Care Survey. J Pain Symptom Manage. 1997;13(2):83-89.

9. Burke A, Upchurch DM, Dye C, Chyu L. Acupuncture use in the United States: findings from the National Health Interview Survey. J Altern Complement Med. 2006;12(7):639-648.

10. Lehmann TR, Russell DW, Spratt KF, Colby H, Liu YK, Fairchild ML, Christensen S. Efficacy of electroacupuncture and TENS in the rehabilitation of chronic low back pain patients. Pain. 1986;26(3):277-290.

11. Ng MM, Leung MC, Poon DM. The effects of electroacupuncture and transcutaneous electrical nerve stimulation on patients with painful osteoarthritic knees: a randomized controlled trial with follow-up evaluation. J Altern Complement Med. 2003;9(5):641-649.

12. Sakai T, Tsutani K. A multicenter randomized trial of electroacupuncture and TENS for low back pain [in Japanese]. Japan Soc Acupunct Moxibustion J. 2001;51(2):175-184.

13. Kuroiwa K. An Approach to Trigger Points for Clinicians [in Japanese]. Tokyo: Idou no Nihonsha; 1999.

14. Green S, Buchbinder R, Hetrick S. Acupuncture for shoulder pain. Cochrane Database Syst Rev. 2005;2:CD005319.

15. Ghoname EA, Craig WF, White PF, Ahmed HE, et al. Percutaneous electrical nerve stimulation for low back pain: a randomized crossover study. JAMA. 1999;281(9):818-823.

16. Tsukayama H, Yamashita H, Amagai H, Tanno Y. Randomised controlled trial comparing the effectiveness of electroacupuncture and TENS for low back pain: a preliminary study for a pragmatic trial. Acupunct Med. 2002;20(4):175-180.
17. Chiu TT, Hui-Chan CW, Chein G. A randomized clinical trial of TENS and exercise for patients with chronic neck pain. Clin Rehabil. 2005;19(8):850-860.

18. Nabeta T, Kawakita K. Relief of chronic neck and shoulder pain by manual acupuncture to tender points-a sham-controlled randomized trial. Complement Ther Med. 2002;10(4): 217-222.

19. Tunis SR, Stryer DB, Clancy CM. Practical clinical trials: increasing the value of clinical research for decision making in clinical and health policy. JAMA. 2003;290(12):1624-1632.

20. Orikasa H. An Introduction to Statistics Used in Clinical Research Design [in Japanese]. Tokyo: Shinko Trading Company; 1995.

21. Melzack R, Stillwell DM, Fox EJ. Trigger points and acupuncture points for pain: correlations and implications. $\underline{\text { Pain }}$. 1977;3(1):3-23.

22. Peng AT, Behar S, Yue SJ. Long-term therapeutic effects of electro-acupuncture for chronic neck and shoulder pain-a double blind study. Acupunct Electrother Res. 1987;12(1): $37-44$.

23. Nakayama T, Hayashi T. The state of TENS therapy. In: Transcutaneous Electrial Nerve Stimulation [in Japanese]. Tokyo: Ishiyaku Publishers; 2011:74-75.

24. Hay EM, Dziedzic K, Sim J. Treatment options for regional musculoskeletal pain: what is the evidence? Baillieres Clin Rheumatol. 1999;13(2):243-259.

25. Fukuhara S. SF-36 Version 2 Japanese Manual [in Japanese]. Tokyo: Institute for Health Outcomes and Process Evaluation Research; 2004.

26. Senn S. Statistical Issues in Drug Development. Chichester, UK: John Wiley \& Sons; 1997.

27. Hills M, Armitage P. The two-period cross-over clinical trial. Br J Clin Pharmacol. 1979;8(1):7-20.

28. Kennedy S, Baxter GD, Kerr DP, Bradbury I, Park J, McDonough SM. Acupuncture for acute non-specific low back pain: a pilot randomised non-penetrating sham controlled trial. Complement Ther Med. 2008;16(3):139-146.

29. Delaney GA, McKee AC. Inter- and intra-rater reliability of the pressure threshold meter in measurement of myofascial trigger point sensitivity. Am J Phys Med Rehabil. 1993;72(3): $136-139$.

30. Reeves JL, Jaeger B, Graff-Radford SB. Reliability of the pressure algometer as a measure of myofascial trigger point sensitivity. Pain. 1986;24(3):313-321.

Address correspondence to:

Alan R. Teo, MD

Robert Wood Johnson Foundation Clinical Scholars Program

University of Michigan

6312 Medical Science Building I

1150 West Medical Center Drive Ann Arbor, MI 48109-0604

E-mail: alan.teo@stanfordalumni.org 